\title{
Electronic Structure of Ligated CdSe Clusters: Dependence on DFT Methodology
}

\author{
Victor V. Albert, ${ }^{\dagger}$ Sergei A. Ivanov, ${ }^{\dagger}$ Sergei Tretiak, ${ }^{\dagger}$ and Svetlana V. Kilina ${ }^{*,+}$ \\ ${ }^{\dagger}$ Theoretical Division and Center for Integrated Nanotechnologies, Los Alamos National Laboratory, Los Alamos, \\ New Mexico 87545, United States \\ ${ }^{\ddagger}$ Department of Chemistry and Biochemistry, North Dakota State University, Fargo, North Dakota 58108, United States
}

Supporting Information

ABSTRACT: Simulations of ligated semiconductor quantum dots (QDs) and their physical properties, such as morphologies, QD-ligand interactions, electronic structures, and optical transitions, are expected to be very sensitive to computational methodology. We utilize Density Functional Theory (DFT) and systematically study how the choice of density functional, atom-localized basis set, and a solvent affects the physical properties of the $\mathrm{Cd}_{33} \mathrm{Se}_{33}$ cluster ligated with a trimethylphosphine oxide ligand. We have found that qualitative performance of all exchange-correlation (XC) functionals is relatively
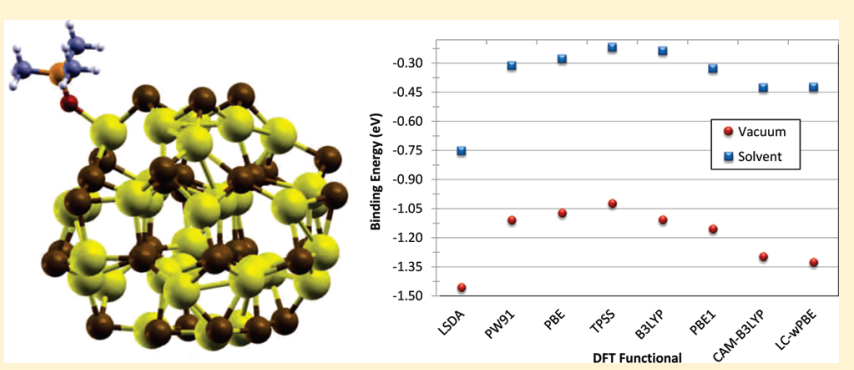
similar in predicting strong QD-ligand binding energy $(\sim 1 \mathrm{eV})$. Additionally, all functionals predict shorter Cd-Se bond lengths on the QD surface than in its core, revealing the nature and degree of QD surface reconstruction. For proper modeling of geometries and QD-ligand interactions, however, augmentation of even a moderately sized basis set with polarization functions (e.g., LANL2DZ* and $\left.6-31 G^{*}\right)$ is very important. A polar solvent has very significant implications for the ligand binding energy, decreasing it to $0.2-0.5 \mathrm{eV}$. However, the solvent model has a minor effect on the optoelectronic properties, resulting in persistent blue shifts up to $\sim 0.3 \mathrm{eV}$ of the low-energy optical transitions. For obtaining reasonable energy gaps and optical transition energies, hybrid XC functionals augmented by a long-range Hartree-Fock orbital exchange have to be applied.

\section{INTRODUCTION}

In many respects, the cadmium selenide colloidal semiconductor nanocrystal (also called CdSe quantum dots, QDs) is a near-ideal material for various applications, including light-emitting devices, ${ }^{1,2}$ solar cells, ${ }^{3}$ quantum computing, ${ }^{4}$ optical amplifiers, ${ }^{5,6}$ and fluorescent biolabels. ${ }^{7,8}$ Sample nanocrystal size can be synthetically controlled resulting in the monodispersed CdSe QDs with a diameter of $2-6 \mathrm{~nm}$. Such dimensions are significantly smaller than the Bohr exciton radius in the bulk material. This leads to a spatial confinement of the electron wave function and results in unique optical and electronic properties. ${ }^{9}$ For example, CdSe QDs demonstrate broad absorption and narrow (pure color) emission spectra, unmatched photostability, and bright luminescence (brightness) with high quantum yield. In addition, the color of their emission can be tuned in a wide range of the visible-light spectrum just by changing their size (see ref 10 for a recent review). However, the main roadblock for practical applications of these characteristics is the high sensitivity of QD optical properties to their surface passivation and chemical environment.

Experiments have established that QDs consist of an inorganic core (the inner atoms), an inorganic surface with a morphology distinct from that of the core, and a shell of organic ligands bonded to the surface. ${ }^{11,12}$ It is supposed that the core determines the physical properties of the QD by its bulk material structure and confinement effects, while a surface layer brings some perturbation to these properties. The ligands protect the surface of the QD during the synthesis, which takes place in a solution comprised of special surfactants. Typically, these are coordinating organic molecules ${ }^{13}$ that remove unsaturated valences (dangling bonds) on the surface of the QD, increase solubility, prevent $\mathrm{QD}-\mathrm{QD}$ interactions, etc. Usage of surface ligands leads to a greater synthetic control over the electrooptical properties of QDs, in particular, improving their emission quantum yield. However, the high surface-to-volume ratio of QDs and imperfect surface passivation, which is mainly determined by the type of ligand, lead to impurities and defects. The latter is likely to add the manifold of additional surface states (trap states) to the electronic structure of the QD and, indeed, affect both radiative and nonradiative processes. Such uncontrolled surface chemistry of QDs may lead to low quantum yield and effects such as bleaching (degradation of luminescence intensity over time) and blinking (fluorescence intermittency). ${ }^{14,15}$ These phenomena are currently holding back usage of QD materials in such potential applications as single-photon light sources for lasers and biolabels.

Received: March 18, 2011

Revised: June 25, 2011 
In addition, the surface chemistry of QDs has a strong influence on relaxation dynamics, i.e., the conversion of the photoexcitation to heat. This influence leads to the absence of the theoretically predicted phonon bottleneck in QDs. ${ }^{16}$ Understanding of surface processes is also important for controlling carrier multiplication (CM), a process in which two or more electron-hole pairs are generated from a single absorbed photon, promising higher efficiency of QD-based solar cells than is currently possible. ${ }^{17}$ Recent time-resolved experiments have demonstrated that radiative quantum yield, ${ }^{18-20}$ exciton lifetime, ${ }^{21}$ relaxation rates, ${ }^{19,22,23}$ and the efficiency of the $\mathrm{CM}^{24}$ are strongly affected by the type of ligands passivating the $\mathrm{QD}$ surface. Various contradicting data on CM efficiency reported in recent literature ${ }^{25-28}$ may also be a result of uncontrolled differences in QD surface structure and treatment. ${ }^{24}$ Thus, the problem of understanding and controlling the several potentially useful functionalities of QDs calls for more rigorous studies of surface passivation and of the role that ligands play in the optoelectronic properties of QDs.

It is experimentally challenging to resolve spectral features originating from $\mathrm{QD}$-ligand interactions, which do not have direct signatures in the conventional spectroscopies. ${ }^{29}$ Theoretical and computational modeling could provide a valuable insight into these issues. However, the lack of periodicity and the relatively large number of atoms make theoretical studies of QDs rather challenging. Commonly used models represent the QD core with tight-binding ${ }^{30,31}$ or pseudopotential ${ }^{32}$ approaches, while the passivating molecules have been modeled through either single-atom ${ }^{30}$ or simplified Gaussian-type potentials. ${ }^{33}$ Any realistic model, however, has to explicitly describe bonding between the QD and the ligand molecules, which is lacking or not complete in the approaches mentioned above. Recently, a few efforts have been done to model ligated CdSe QDs using force field ${ }^{34,35}$ and Monte Carlo methods. ${ }^{38}$ These methods, however, are based on a model description of the molecular framework and interactions. Such parameters are unavailable from measurements, making the accuracy of these approaches problematic. To overcome this problem, the model parameters reported in refs 35 and 36 are derived from Density Functional Theory (DFT) applied to small CdSe clusters.

First-principle calculations such as DFT methods are able to provide the information on bonding and electronic structure with a reasonable degree of accuracy. Unfortunately, DFT is numerically expensive, so most DFT calculations have been performed on small CdSe clusters of a few atoms in size with or without capping ligands. Calculations focusing on the structural and optical properties of small CdSe clusters were usually performed with hybrid exchange-correlation (XC) functionals such as B3LYP and with the LANL2DZ basis set, utilizing either time-dependent DFT (TD-DFT) ${ }^{37-40}$ or symmetry adapted coupled cluster theory with configuration interaction (SAC$\mathrm{CI})^{41}$ for simulations of absorption spectra. Structures of larger CdSe clusters (1.3-2 $\mathrm{nm}$ in diameter) have been simulated with the local density approximation (LDA) using a real space grid ${ }^{42}$ and generalized gradient approximation (GGA) functionals coupled with plane-wave basis sets. ${ }^{43-48}$ On the basis of these methods, a few efforts have been made to address the specifics of the electronic structure and binding energies of CdSe QDs interacting with a single ligand molecule ${ }^{44,45}$ and with many ligands, fully or partially passivating the surface of the $\mathrm{Cd}_{33} \mathrm{Se}_{33} \mathrm{QD}$. ${ }^{48}$

As mentioned above, various XC functionals and basis sets have been utilized to simulate QD-ligand structures. However, systematic analyses and comparisons of the methodologies implemented in simulations (in particular, performance of various XC functionals and basis sets for QD-ligand interactions, electronic structures, and optical spectra) have not been carried out to date, except for very small clusters consisting of a few atoms. ${ }^{36,37}$ The results of ref 37 suggest that B3LYP/ LANL2DZ, the mostly widely used functional/basis set combination for simulations of QDs, is appropriate for modeling ligated CdSe clusters, while superposition error, zero-point energy, and solvent effects produce minor variations in the ligand-cluster interactions. In contrast, the LANL2DZ basis set was found to overestimate $\mathrm{Cd}-\mathrm{Se}$ bond distances, providing an improper description of the structure and energetics of the clusters, as reported in ref 36. This calls for further investigations of the validity of the DFT methodologies for larger CdSe clusters, which are expected to be better models of realistic systems.

In this article, we systematically study how the QD-ligand binding energy, bond length, electronic structure, and the lowest excitation energy depend on the choice of basis set, functional, and solvent model in a $\mathrm{Cd}_{33} \mathrm{Se}_{33}$ cluster interacting with a single trimethyl-phosphine oxide $\left(\mathrm{OPMe}_{3}\right)$ ligand. Although various acidic phosphorus species in addition to (or instead of) trioctylphosphine oxide (TOPO) ligands have been recently discovered to play an important role in passivating the surface of colloidal QDs, ${ }^{49-52}$ we chose $\mathrm{OPMe}_{3}$ - a reduced model of TOPO-as the baseline to compare our results to previous theoretical investigations. ${ }^{44,45,48}$ As expected, all the aforementioned properties are method-sensitive. We found that the choice of a relatively flexible basis set with polarization functions (e.g., $6-31 \mathrm{G}^{*}$ or LANL2DZ*) included for ligand atoms (at least) is crucial for accurate modeling of geometries and QD-ligand interactions. Using functionals with the long-range orbital exchange (hybrid kernel) is important for obtaining a plausible description of QD-ligand bonding and optical transition energies comparable to the experimental values. Inclusion of a polarizable continuum model accounting for a solvent environment in calculations significantly reduces QD-ligand binding energy values. However, it has only a minor effect on the energy gap and transition energies. Overall, depending on the specific physical properties that are modeled, a specific functional/basis set combination has to be chosen for proper simulations of the ligated QDs.

\section{COMPUTATIONAL DETAILS}

All calculations have been performed using the Gaussian09 software package. ${ }^{53}$ The initial QD cluster was constructed out of the wurtzite lattice with bulk $\mathrm{Cd}-\mathrm{Se}$ bond lengths, and then its geometry was optimized to the lowest-energy configuration (see Figure 1). The QD-ligand binding energy between the CdSe cluster and the $\mathrm{OPMe}_{3}$ ligand is defined as a difference between the total energy of the optimized cluster with the adsorbed ligand and the total energies of the optimized bare $\mathrm{QD}$ and the isolated ligand. All geometry optimizations and subsequent calculations of the electronic structure are performed using Kohn-Sham (KS) theory implementing different functionals with and without solvent effects. Several types of functionals are considered, representing different choices of the exchange-correlation kernel. The semilocal functionals used include the Local Spin Density Approximation (LSDA), the Generalized Gradient Approximations (GGA) in the form of $\mathrm{PBE}^{54}$ and $\mathrm{PW} 91^{55}$ models, and the newest TPSS functional representing the meta-Generalized Gradient Approximation. ${ }^{56}$ Semilocal functionals can be constructed from first principles either without empirical parametrization, such 


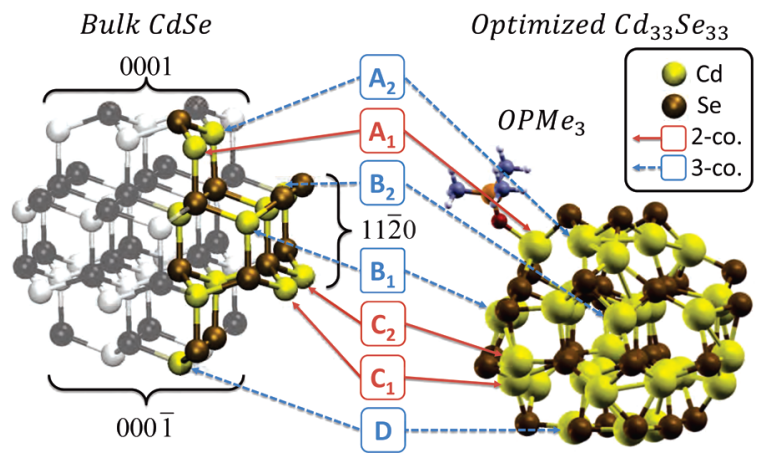

Figure 1. Structure of the calculated $\mathrm{Cd}_{33} \mathrm{Se}_{33}$ cluster. Left panel shows the initial configuration obtained from bulk CdSe crystal. One-third of the crystal is in color, highlighting its 3-fold symmetry. Two- and three-coordinated atoms on the surface of the cluster are listed in red and blue, respectively. Miller-Bravais indices for surfaces of the cluster are given in black. Right panel shows the optimized structure of the $\mathrm{Cd}_{33} \mathrm{Se}_{33}$ functionalized with a single trimethylphosphine oxide $\left(\mathrm{OPMe}_{3}\right)$ ligand at the position $\mathrm{A}_{1}$. Optimization leads to a reconstruction of the surface such that all surface atoms become at least 3-coordinated.

as LDA, PBE, and TPSS, or with some empirically chosen constraints, such as PW91. We also use several hybrid functionals, which add a fraction (A) of nonlocal Hartree-Fock (HF) exchange to the exchange-correlation kernel. ${ }^{57}$ Here, we have chosen several commonly used functionals of this type: B3LYP $(A=20 \%), \operatorname{PBE}^{58}(A=25 \%)$, CAM-B3LYP ${ }^{59}(A=20-65 \%)$, and $\mathrm{LC}-\omega \mathrm{PBE}^{60}(A=0-100 \%)$. The last two models represent long-range-corrected functionals, which behave as a typical hybrid or GGA at short range. However, they have an increasing HF component at longer distances up to a maximum value. The excited states (optical transitions) have been studied using the linear response TD-DFT formalism, ${ }^{61}$ in which the adiabatic approximation for the $\mathrm{XC}$ kernel is used, with the same functionals as described above.

For the $\mathrm{QD}$ atoms ( $\mathrm{Cd}$ and $\mathrm{Se}$ ), we use the relativistically corrected Effective Core Potential (EPS) double- $\zeta$ quality LANL2DZ basis set (denoted as L in Figure 2). For the lighter elements (except $\mathrm{P}$ ), this basis is equivalent to the $\mathrm{D} 95 \mathrm{~V}$ basis. We also augment this basis with extra polarization functions ( $d$ for $\mathrm{H}, \mathrm{C}$, and $\mathrm{O}$ elements and $f$ for $\mathrm{P}, \mathrm{Cd}$, and $\mathrm{Se}$ ) and have denoted it as LANL2DZ* (abbreviated as $\mathrm{L}^{*}$ in Figure 2). We have also applied the Def2-TZVP basis set (abbreviated as d2-tzvp in Figure 2) - the largest basis set we consider here. For the ligand atoms, we have verified the application of both LANL2DZ and LANL2DZ* sets. In addition, basis sets more commonly used for organic molecules, such as 6-31G, 6-311G, as well as their versions augmented with additional diffuse $(+)$ and polarization $\left(^{*}\right)$ functions, have been tested. In our notation, the basis sets used for the QD and ligand atoms are separated with a "/" with the QD's basis set being written first. For example, L/6-31G means that LANL2DZ is used for Cd and Se atoms in the QD and the 6-31G basis set is applied for all ligand atoms $(\mathrm{O}, \mathrm{P}, \mathrm{C}$, and $\mathrm{H})$. For comparison, we have also performed the geometry optimization using the PW91 functional and a plane-wave basis set coupled with the Vanderbilt pseudopotentials, ${ }^{62}$ as incorporated in the VASP computational package. ${ }^{63}$ The simulations are carried out in a cubic cell periodically replicated in three dimensions with $8 \AA$ of vacuum between the periodic images, in accordance with similar calculations. ${ }^{44,48}$

Finally, solvent effects are simulated by embedding the molecule in a polarizable continuum medium with an appropriate

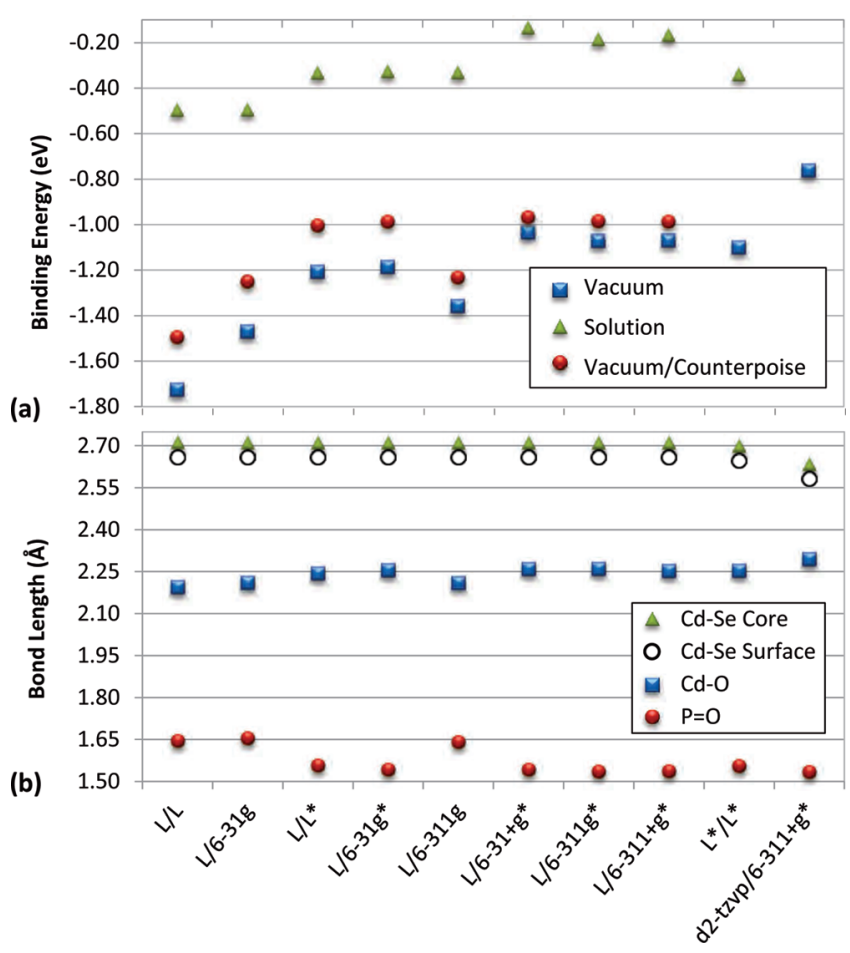

Figure 2. Metal-ligand binding energies (a) and bond lengths (b) plotted vs basis set used. All geometries of $\mathrm{Cd}_{33} \mathrm{Se}_{33}$ functionalized with $\mathrm{OPMe}_{3}$ are optimized using the B3LYP functional. In notations, the basis set for $\mathrm{Cd}$ and Se atoms is listed first, while the basis set for the ligand atoms is listed second and separated with a "/". For brevity, the symbols "L" and "d2-tzvp" stand for LANL2DZ and Def2-TZVP basis sets, respectively. The symbol " $\mathrm{L}^{*}$ " stands for the LANL2DZ", which includes extra polarization functions in the basis set, which are important for an accurate description of $\mathrm{QD}$ - ligand binding and $\mathrm{Cd}-\mathrm{O}$ and $\mathrm{P}-\mathrm{O}$ bond lengths. The $\mathrm{CP}$ correction noticeably alters the performance of relatively small basis sets, while expectedly it has a small effect on the extended basis sets. Solvent noticeably weakens QD-ligand interaction.

dielectric constant in the framework of the polarizable conductor calculation model (CPCM), ${ }^{64,65}$ as implemented in Gaussian09. Acetone $(\varepsilon=20.7)$, used frequently in the relevant experimental studies, has been chosen as a solvent.

\section{RESULTS AND DISCUSSION}

The $\mathrm{Cd}_{33} \mathrm{Se}_{33}$ "magic" structure with a diameter of $1.3 \mathrm{~nm}$ is the smallest cluster that supports a crystalline-like core. ${ }^{66}$ It is expected to have good chemical stability ${ }^{67}$ and efficient blue-light emission. ${ }^{68}$ This makes $\mathrm{Cd}_{33} \mathrm{Se}_{33}$ an excellent model for studying both the solid-state and molecular properties of QDs. In accordance with previous simulations, ${ }^{48,44}$ we constructed the $\mathrm{Cd}_{33} \mathrm{Se}_{33}$ from a wurtzite lattice with bulk $\mathrm{Cd}-\mathrm{Se}$ bond lengths. As was discussed in the previous computational ${ }^{37,45,48}$ and experimental ${ }^{20}$ studies, the dominant binding interactions occur between the oxygen of the ligand and $\mathrm{Cd}$ atom of the QD surface. Therefore, we attach the $\mathrm{OPMe}_{3}$ ligand to the cadmium atom on one of the surface sites marked as A, B, C, and D in Figure 1. The initial bulk-like and the relaxed structures of the $\mathrm{Cd}_{33} \mathrm{Se}_{33}$ covalently bound with $\mathrm{OPMe}_{3}$ at the site $\mathrm{A}$ are illustrated in Figure 1.

In the bulk (unrelaxed) geometry of $\mathrm{Cd}_{33} \mathrm{Se}_{33}$, there are two types of surface atoms: 2 -coordinated atoms that are bound to only two adjacent $\mathrm{Se}$ atoms, thus having two unsaturated valences 
Table 1. Metal-ligand Binding Energies (eV) of the $\mathrm{OPMe}_{3}$ with $\mathrm{Cd}_{33} \mathrm{Se}_{33}$ at Each of the Nonsymmetry-Related Surface Sites: Comparison of Our Calculations with the Published Data of Similar Systems

\begin{tabular}{|c|c|c|c|c|c|c|c|}
\hline \multirow[b]{2}{*}{ method } & \multicolumn{2}{|c|}{$(000 \overline{1})$} & \multicolumn{3}{|c|}{$(11 \overline{2} 0)$ and $(01 \overline{1} 0)$} & \multirow{2}{*}{$\frac{(0001)}{\mathrm{D}}$} & \\
\hline & $A_{1}$ & $\mathrm{~A}_{2}$ & B & $\mathrm{C}_{1}$ & $\mathrm{C}_{2}$ & & \\
\hline B3LYP/(LANL2DZ/631G) & -1.45 & -1.20 & -1.12 & -1.42 & -1.39 & -1.05 & this work \\
\hline PW91/(plane waves) & $-0.76(-0.61)$ & -0.73 & -0.46 & -1.37( & & -0.23 & $\operatorname{ref} 48^{a}$ \\
\hline LDA/(plane waves) & -0.85 & & -1.23 & & & -0.63 & ref 44 \\
\hline PW91/(plane waves) & -0.31 & & -0.51 & & & 0.03 & $\operatorname{ref} 45^{b}$ \\
\hline
\end{tabular}

${ }^{a}$ In ref 48, several $\mathrm{OPMe}_{3}$ molecules on the $\mathrm{Cd}_{33} \mathrm{Se}_{33}$ are considered: full passivation of all cadmiums on the surface and partial passivation of only 2-coordinated Cd atoms (shown in parentheses). ${ }^{b}$ In ref 45, adsorption of the $\mathrm{OPH}_{3}$ on the CdSe bulk surface is considered.

(marked by red in Figure 1), and 3-coordinated atoms that are bound to three adjacent Se atoms, having one unsaturated valence (marked by blue in Figure 1). Upon optimization, the system surface "self-heals" by rearranging its atoms so that the 2 -coordinated $\mathrm{Cd}$ and Se atoms become 3-coordinated. Surface reconstruction reduces the symmetry of the crystal: four nonsymmetryequivalent facets corresponding to Cd-terminated (0001) and Seterminated $(11 \overline{2} 0,01 \overline{1} 0$, and 0001$)$ surfaces partially break their symmetry, and atoms on these surfaces can now be identified as $A_{1}$ and $A_{2}, B, C_{1}$ and $C_{2}$, and D, as illustrated in Figure 1. Such surface relaxations were shown to contribute to an increase in the band gap of CdSe clusters. ${ }^{43,44,47,48}$

Dependence of QD-Ligand Interaction Strength on Ligand Placement. The binding energies characterizing the strength of the interaction between the $\mathrm{QD}$ surface and the ligand at each of the sites $\left(A_{1}, A_{2}, B, C_{1}, C_{2}\right.$, and $\left.D\right)$ are presented in Table 1. To obtain these data, we have optimized structures using the B3LYP functional and LANL2DZ basis set for Cd and Se atoms of the QD and 6-31G basis set associated with ligand atoms (LANL2DZ/6-31G). As expected, the strongest ligand binding energy is found to be with the most chemically active, 2-coordinated $\mathrm{Cd}$ atoms $\left(\mathrm{A}_{1}, \mathrm{C}_{1}\right.$, and $\left.\mathrm{C}_{2}\right)$, which agrees well with previous calculations. ${ }^{44,48}$ The weakest interaction is observed at the $\mathrm{D}$ site corresponding to the $(000 \overline{1})$ Cd-terminated surface. Qualitatively, these results agree well with earlier results reported

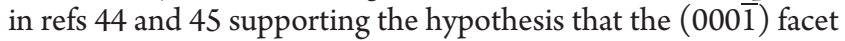
likely experiences preferential growth because of its predominantly unpassivated character and, thus, controls the formation of 1-D nanocrystals (nanorods).

However, the obtained values are noticeably larger (in absolute value) that those reported in other works. There are several reasons for this discrepancy. First, our calculations do not include intermolecular interactions between neighboring ligands on the surface, which were included in ref 48 . As expected, the interligand interactions decrease the binding energy. Second, the finite size and shape of the QD has a strong effect on the QD-ligand interactions, resulting in larger absolute values of the binding energies compared to those of the CdSe bulk surface. ${ }^{45}$ Lastly, we have observed the binding energies to be sensitive to the choice of underlying theoretical methodology and solvent model.

To estimate the sensitivity of these properties with respect to the chosen computational method, we have performed a set of calculations of the $\mathrm{Cd}_{33} \mathrm{Se}_{33}$ cluster ligated with $\mathrm{OPMe}_{3}$ using different functionals and various atom-localized basis sets. For a uniform comparison, we have chosen only one ligand binding site $A_{1}$. This position provides very stable $Q D$-ligand interactions (Table 1), while preserving its symmetry as the Cd-terminated
(0001) facet in bulk material. It also allows for comparison of our results with previously reported calculations. ${ }^{44,45}$

Effect of Basis Sets on Ligand Binding to the QD. The dependence of the binding energy on the basis set in vacuum and in the presence of acetone solvent is shown in Figure 2(a) for $\mathrm{Cd}_{33} \mathrm{Se}_{33}$ passivated by OPMe $\mathrm{O}_{3}$ at the site $\mathrm{A}_{1}$. The B3LYP hybrid density functional is used for all calculations presented in Figure 2. Evidently, the presence of extra polarization functions in the basis set associated with ligand atoms is very important for the proper description of the $\mathrm{QD}$-ligand interaction. When polarization functions are added (LANL2DZ/LANL2DZ*, LANL2DZ/6-31G*, and LANL2DZ/6-311G*), the absolute value of the binding energy decreases by about $0.3-0.5 \mathrm{eV}$. The performances of LANL2DZ/LANL2DZ* and LANL2DZ/ 6-31G* are very similar: both result in a binding energy of $-1.2 \mathrm{eV}$. However, compared to the latter, the LANL2DZ/ LANL2DZ* approach is numerically less expensive, making this basis set more beneficial for similar types of calculations.

The diffuse functions in the $6-31+G^{*}$ basis set lead to a further decrease of the QD-ligand interaction of about $0.2 \mathrm{eV}$. It is worth noting that the inclusion of the diffuse functions in the 6-31G basis set used for the organic ligand atoms (LANL2DZ/6$\left.31+G^{*}\right)$ has a similar effect on the binding energy as the use of more extended basis sets LANL2DZ/6-311G*, LANL2DZ/6$311+\mathrm{G}^{*}$, and LANL2DZ*/LANL2DZ*. In the next-to-last entry in Figure 2(a), the LANL2DZ basis set with polarization functions is used for both QD and organics. In all these cases, the binding energy reaches a value of about $-1.0 \mathrm{eV}$. Additional augmentation of the basis set (Def2-TZVP/6-311+G*) leads to even further decrease of the $\mathrm{QD}$-ligand interactions, predicting the binding energy of about $-0.8 \mathrm{eV}$. This value is in good agreement with similar calculations performed with the LDA functional and the plane-wave basis set, ${ }^{44}$ listed in Table 1. Our calculations based on the PW91 functional with the plane-wave basis set resulted in a binding energy of $-0.87 \mathrm{eV}$. The counterpoise correction $(\mathrm{CP})$ adjusts the ligand binding energy by $\sim 0.2$ $\mathrm{eV}$ for the relatively small basis sets but has a little effect $(\sim 0.06$ $\mathrm{eV}$ ) for larger basis sets. Thus, the CP correction allows one to obtain fairly accurate binding energies with relatively small basis sets such as LANL2DZ/LANL2DZ* or LANL2DZ/6-31G*. Unfortunately, its evaluation is often almost as expensive computationally as the use of a more extended basis set.

A polar solvent has been found to have very significant effects on the ligand binding energy (Figure 2). Incorporation of an acetone solvent environment into our calculations results in much smaller QD-ligand interactions: the ligand binding energies in solvent vary between -0.5 and $-0.2 \mathrm{eV}$, depending on the basis set. Thus, the polar solvent screens the QD-ligand 
interaction and makes it $\sim 70 \%$ weaker compared to the respective gas-phase values. Such strong effects likely originate from the large dipole moments of the CdSe $\mathrm{QDs}^{69}$ and $\mathrm{OPMe}_{3}$ ligands, which are expected to affect the binding and, indeed, should be screened by the polar solvent. In smaller clusters of a few atoms in size, the solvent effect was found to result in small, less than $10 \%$, changes in ligand binding to the $\mathrm{Cd}_{2} \mathrm{Se}_{2}$ cluster in chloroform solution. ${ }^{37}$ This discrepancy can be attributed to nonpolar character of the chloroform, as well as to a reduced dipole moment of the symmetric $\mathrm{Cd}_{2} \mathrm{Se}_{2}$ molecule. ${ }^{69}$

Despite a significant decrease in the QD-ligand interaction in the polar media, the values of the binding energy are still much larger than thermal fluctuations, so that covalently bound ligands should dominate over the physisorbed molecules in experimental samples of QDs. The overall trend of the binding energy dependence on the basis sets in solvent is similar to that in vacuum, with the exception of basis sets without polarization functions. For LANL2DZ/LANL2DZ, LANL2DZ/6-31G, and LANL2DZ/6-311G, the binding energy is slightly corrected by the presence of the solvent, making it more consistent with the results obtained by the basis sets with polarization functions.

Figure 2(b) presents the dependence of the geometry of the ligated $\mathrm{QD}$ on the basis sets. The $\mathrm{Cd}-\mathrm{O}$ bond length correlates well with the strength of the QD-ligand interaction: it is longer $(2.25-2.28 \AA)$ for weaker $\mathrm{QD}$-ligand interactions and shorter $(\sim 2.20 \AA)$ for stronger QD-ligand binding. Therefore, the increase of the $\mathrm{Cd}-\mathrm{O}$ bond length with the basis set augmentation is concomitant with the trends in the binding energy. The $\mathrm{Cd}-\mathrm{Se}$ bonds are found to be the least sensitive to the basis set size. Nonetheless, they become shorter (by $\sim 0.08 \AA$ ) when a very flexible basis set, such as Def2-TZVP/6-311+G*, is used. The Cd-Se bonds of $2.62 \AA$ in the QD core and $2.58 \AA$ on the surface obtained from the Def2-TZVP/6-311+G* basis set correlate well with results of cc-pVTZ-SD*, VDZ-SD*, and $\operatorname{SBKJ}(\mathrm{d} / 2 \mathrm{df})$ basis sets applied to smaller $\mathrm{Cd}_{n} \mathrm{Se}_{n}(n=2,3, \ldots$, 16) clusters. $^{36}$ All other basis sets we consider slightly overestimate the $\mathrm{Cd}-\mathrm{Se}$ bonds, predicting $\sim 2.70 \AA$ in the $\mathrm{QD}$ core and $\sim 2.66 \AA$ on the surface. As expected, the length of the $\mathrm{P}-\mathrm{O}$ bond is very sensitive to the basis set size and, in particular, to the presence of polarization functions. When polarization functions are added to the basis, the $\mathrm{P}-\mathrm{O}$ bond decreases from $1.63-1.64$ to $1.52-1.54 \AA$.

Overall, both geometries and binding energies of the ligated CdSe QDs are dependent on basis set size. To improve computational accuracy, polarization functions have to be added at least to the part of the basis set associated with the organic ligand atoms. Thus, to obtain qualitatively accurate geometries and QD-ligand interactions, the smallest basis set has to be either LANL2DZ/LANL2DZ* or LANL2DZ/6-31G*. Both have very similar performance, which can be quantitatively improved by adding the $\mathrm{CP}$ correction or supplementing a polarization function to the basis set of the QD. For quantitatively correct values of the binding energies and bond lengths, however, the more extended basis sets need to be chosen (e.g., Def2-TZVP/6$\left.311+\mathrm{G}^{*}\right)$. More importantly, incorporation of the solvent is absolutely necessary in obtaining QD-ligand interactions consistent with experimental data.

Effect of Functionals on Ligand Binding to the QD. In addition to the dependence on the basis set, QD-ligand interactions are expected to be sensitive to the choice of $\mathrm{XC}$ functional. Figure 3(a) shows dependence of the binding energy on the functional in vacuum and in acetone. For all calculations

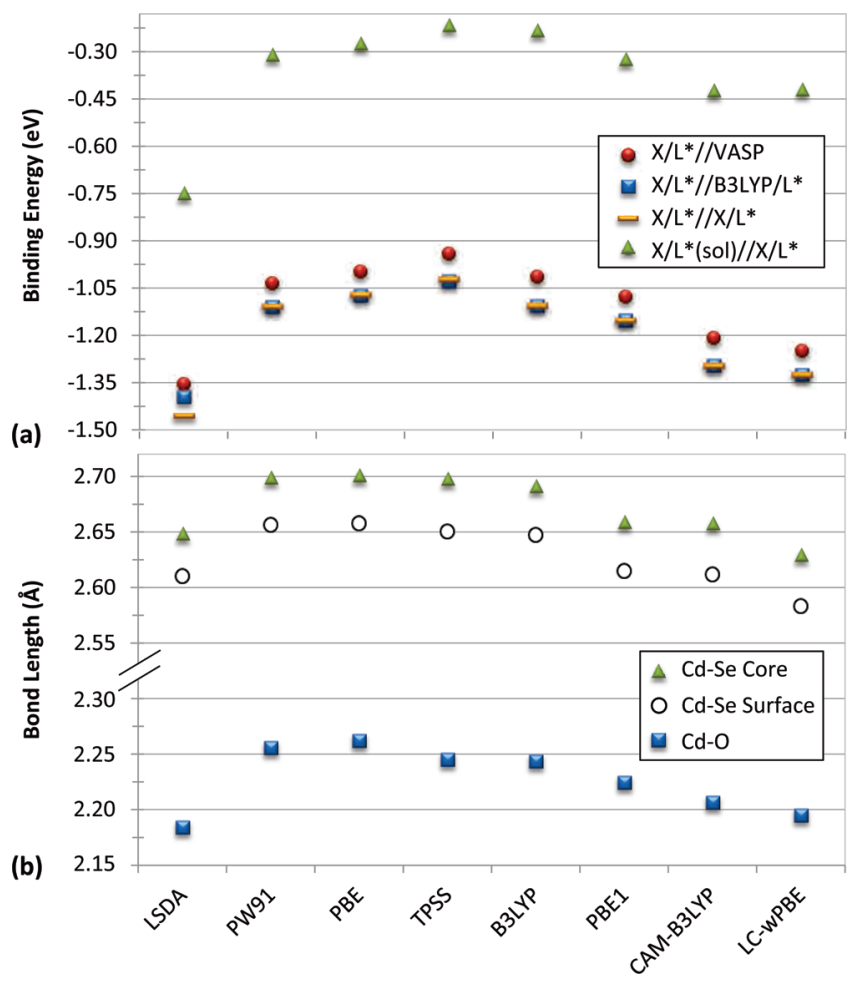

Figure 3. Metal-ligand binding energies (a) and bond lengths (b) plotted vs functional used in calculations of $\mathrm{Cd}_{33} \mathrm{Se}_{33}$ with $\mathrm{OPMe}_{3}$ ligand. All calculations are done with the LANL2DZ* basis set. The first part before the "//" stands for the functional and basis set used for the electronic structure calculations, and the second part corresponds to the functional and basis set used during optimization. For example, $\mathrm{X} / \mathrm{L}^{*} / /$ $\mathrm{B} 3 \mathrm{LYP} / \mathrm{L}^{*}$ means that the system was optimized with B3LYP/ LANL2DZ*, and then its energy was calculated with X/LANL2DZ*, where $\mathrm{X}$ is one of the functionals on the horizontal axis. The abbreviation "VASP" stands for the PW91 functional and a plane-wave basis set used in the VASP computational package. The abbreviation "sol" stands for calculations in acetone. The bond lengths reported in panel (b) are taken from the $\mathrm{X} / \mathrm{L}^{*} / / \mathrm{X} / \mathrm{L}^{*}$ calculations.

shown in Figure 3, LANL2DZ* is used for both the QD and ligand atoms (LANL2DZ*/LANL2DZ*) because of its balance between accuracy and computational efficiency. As shown in Figure 3(a), the LSDA predicts the strongest interaction between the $\mathrm{Cd}_{33} \mathrm{Se}_{33}$ and $\mathrm{OPMe}_{3}$ with a binding energy of about $-1.4 \mathrm{eV}$. This is expected, since a well-known drawback of LSDA is overestimation of the binding energy in many molecular compounds. GGA functionals (PW91 and PBE) and meta-GGA (TPAA) decrease the ligand interaction by $25-30 \%$. Hybrid functionals with a small portion of HF exchange (B3LYP and PBE1) also decrease QD-ligand interactions by about $25 \%$. Among all functionals considered, the TPSS model predicts the weakest binding energy of $-1.0 \mathrm{eV}$. The larger presence of $\mathrm{HF}$ exchange in the long-range corrected functionals CAM-B3LYP and LC- $\omega$ PBE leads to a slight increase in QD-ligand interaction compared to GGA values, which is still about $10 \%$ smaller than the binding energy predicted by the LSDA.

We also calculate the binding energies using various functionals, but with geometries obtained either with the B3LYP functional and LANL2DZ* basis set or with the PW91 functional and a plane-wave basis set, as compared in Figure 3(a). Application of geometries obtained from the optimization by a different 

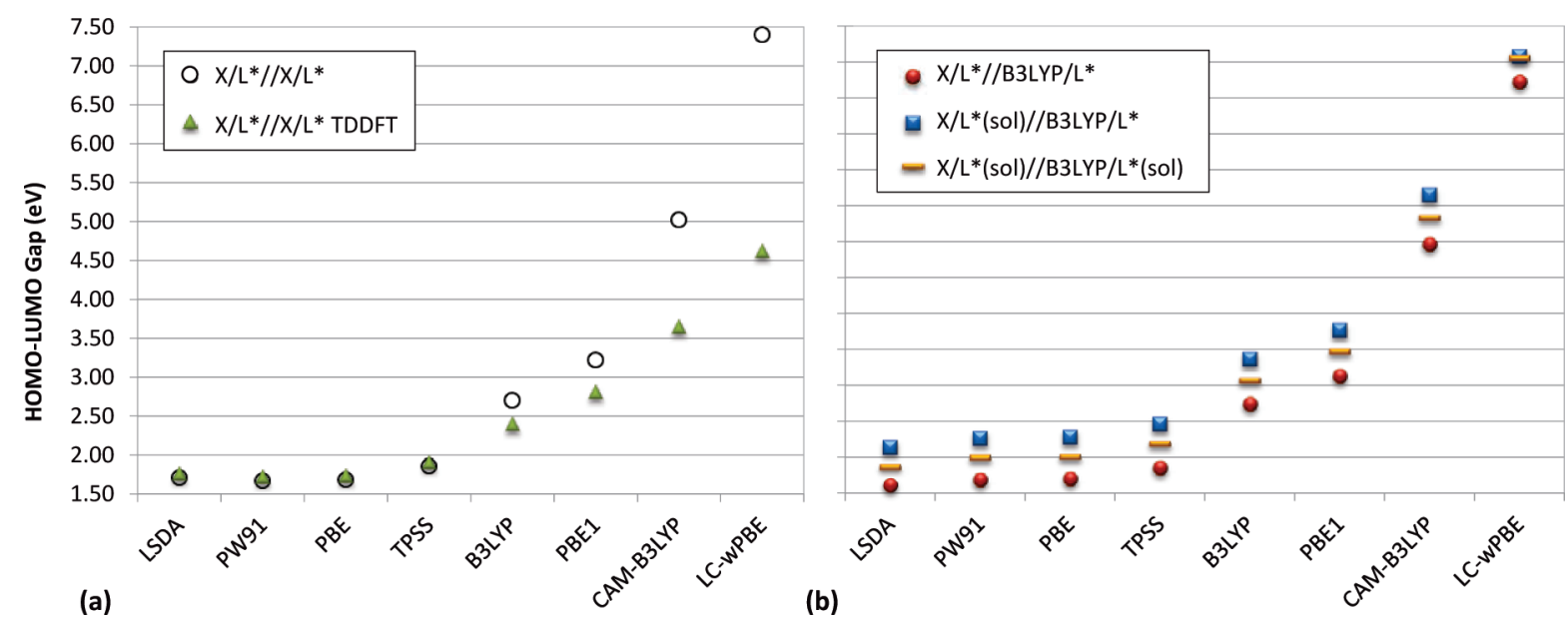

Figure 4. Energy gaps (HOMO-LUMO gaps) and the lowest-energy optical transition of the $\mathrm{Cd}_{33} \mathrm{Se}_{33}$ with the OPMe${ }_{3}$ ligand. Notations are the same as in Figure 3. In panel (a), "TDDFT" stands for the lowest-energy optical transition as calculated by TD-DFT. (b) Comparison of the HOMO-LUMO energy gap calculated in vacuum and in the acetone solvent. Incorporation of the solvent model into calculations slightly blue-shifts the energy gap independent on the functional model.

method, rather than from the method used for the binding energy calculations, has a minor effect on the QD-ligand interactions. While formally incorrect, such an approach provides qualitative evaluation of the binding energies if the method-"native" geometries are not available. As we have already discussed, the presence of solvent strongly decreases the QD-ligand interactions, resulting in binding energies ranging from -0.8 to $-0.2 \mathrm{eV}$, depending on the functional. Qualitatively, however, the dependence of binding energy on the functional in the presence of solvent has the same behavior as that in the gas phase.

The trends observed in binding energies are also reflected in the geometries of the clusters optimized with different functional models. The stronger the $\mathrm{QD}$-ligand interaction, the shorter the metal-ligand bond becomes. As a result, the effect of the functional on the $\mathrm{Cd}-\mathrm{O}$ bonds between the $\mathrm{QD}$ and the ligand, as well as on the $\mathrm{Cd}-\mathrm{Se}$ bonds inside the $\mathrm{QD}$, is well correlated with the binding energy. The effect of the XC functional on the $\mathrm{P}-\mathrm{O}$ bond is the least pronounced: LSDA and GGA functionals slightly increase the $\mathrm{P}-\mathrm{O}$ bond by $\sim 0.02 \AA$, compared to the bond length of $\sim 1.54 \AA$ obtained from hybrid and long range corrected functionals. Overall, the values for the bond lengths provided by the long-range corrected functionals are in very good agreement with results obtained by a more accurate level of theory, coupledcluster with singlets and doublets (CCSD), reported for ligated $\mathrm{Cd}_{2} \mathrm{Se}_{2}$ systems. ${ }^{37}$ Qualitatively, all functionals predict the core $\mathrm{Cd}-\mathrm{Se}$ bond length to be about $0.04 \AA$ longer than the surface bond. The difference between the core and the surface morphologies points to a qualitatively similar and functional independent geometry reorganization mechanism during QD surfece relaxation. Compared to the basis set dependence, the effect of the functional model on the QD-ligand binding is weaker, which agrees with conclusions reported for ligated $\mathrm{Cd}_{2} \mathrm{Se}_{2}$ clusters. $^{37}$

Effect of Exchange-Correlation Functionals on Optical Properties. It is well-known that the choice of functional is very important for an accurate description of the band gap energies and optical spectra of materials in the solid state. For example, because of the electron self-interaction problem, LSDA and GGA functionals usually significantly underestimate the band gap and, consequently, the energy of the optically active transitions, while
HF-like approaches overestimate their magnitudes. ${ }^{70}$ Figure 4 demonstrates the functional dependence of the molecular HOMO-LUMO gap (energy gap) and the lowest optical transition of $\mathrm{Cd}_{33} \mathrm{Se}_{33}$ ligated with $\mathrm{OPMe}_{3}$. As expected, LDA and GGA functionals predict an unrealistically narrow energy gap $(1.68-1.71 \mathrm{eV})$ for such small clusters. When the GGA functionals are corrected by a small portion of HF exchange, i.e., B3LYP and PBE1, the energy gap immediately increases to $\sim 2.7-3.2 \mathrm{eV}$. These values serendipitously agree with the lowest absorption peak $(\sim 2.99 \mathrm{eV})$ experimentally observed and assigned to the $\mathrm{Cd}_{33} \mathrm{Se}_{33} \mathrm{QD}{ }^{67,68}$ Long-range corrected functionals CAM-B3LYP and LC- $\omega$ PBE overestimate the band gap, predicting it to be larger than $5 \mathrm{eV}$. However, when Coulombic interactions between the photoexcited electron and hole are included in these functionals via TD-DFT calculations, the energy of the lowest transition significantly decreases (by $1.5-2 \mathrm{eV}$ ) and becomes closer to the values obtained from standard hybrid functionals. The corrections included in TDB3LYP and TD-PBE1 methods only slightly decrease the energy of the lowest transition by about $0.3-0.4 \mathrm{eV}$, compared to the uncorrelated HOMO-LUMO gap. As expected, TD-DFT corrections do not affect results of LDA and GGA functionals: the energy of the uncorrelated HOMO-LUMO gap and the first optical transition almost coincide. The difference in the behavior of the hybrid and pure XC functionals at the TDDFT level is attributed to a lack of excitonic effects in LSDA and GGA functionals, as have been discussed in a general case in ref 71 and in specific applications of these methods to QDs, ${ }^{42,72}$ conjugated polymers, ${ }^{70}$ and carbon nanotubes. ${ }^{73}$

Figure 4(b) compares the HOMO-LUMO gaps calculated by different functionals in vacuum and in acetone solvent. All calculations are based on the geometries obtained from the B3LYP/LANL2DZ* approach, either in vacuum or in acetone. For all functionals, the solvent blue-shifts the band gaps by about $0.5-0.6 \mathrm{eV}$. This blue shift correlates well with results obtained for small CdSe clusters of a few atoms in size. ${ }^{40}$ When both the electronic structure and geometry optimization have been performed in solvent, the blue shift decreases, and the difference between the band gaps calculated in vacuum and in acetone becomes about $0.2-0.3 \mathrm{eV}$. Overall, the solvent has a small effect 
on the HOMO-LUMO gaps of the ligated CdSe cluster, increasing the gap energies by a maximum of $0.3 \mathrm{eV}$ across the board, regardless of the functional used. A similar blue shift has been found for the lowest-energy transition in the presence of solvent for small CdSe clusters.

\section{CONCLUSIONS}

In summary, DFT and TD-DFT calculations of the $\mathrm{Cd}_{33} \mathrm{Se}_{33}$ cluster with a covalently bound $\mathrm{OPMe}_{3}$ ligand are performed using different atom-localized basis sets and functionals in vacuum and in solvent. We have analyzed sensitivity of geometry, QD-ligand interaction, band gap, and the lowest-energy optical transition of this system with respect to the underlying theoretical method.

We have shown that both geometries and QD-ligand binding energies computed for the ligated CdSe cluster are strongly dependent on basis set size: excluding a few special cases, the binding energy generally decreases with increasing basis size. Inclusion of polarization functions at least to the part of the basis set associated with ligand atoms is crucial for obtaining these properties with reasonable accuracy. Thus, to obtain qualitatively accurate geometries and $\mathrm{QD}$-ligand interactions, the minimal basis set has to be either LANL2DZ/LANL2DZ* or LANL2DZ/ $6-31 G^{*}$. Both have similar performance which can be quantitatively improved by adding the counterpoise correction or/and extra polarization functions to the basis set of the QD (LANL2DZ*/LANL2DZ* or LANL2DZ*/6-31G*). To compute quantitatively correct values of the binding energies, further extension of the basis set size (e.g., Def2-TZVP $/ 6-311^{+} \mathrm{G}^{*}$ ) is suggested.

In general, weaker dependence on the functional model is observed for the binding energy. The LSDA overestimates the QD-ligand interactions and underestimates the length of $\mathrm{Cd}-$ Se bonds in the $\mathrm{QD}$ and $\mathrm{Cd}-\mathrm{O}$ bonds between the $\mathrm{QD}$ and the ligand, when compared to other functionals we consider. GGA (PW91 and PBE), meta-GGA (TPSS), and hybrid functionals with a small portion of HF exchange (B3LYP and PBE1) predict roughly similar $\mathrm{QD}-$ ligand interaction and $\mathrm{Cd}-\mathrm{Se}$ and $\mathrm{Cd}-\mathrm{O}$ bond lengths. Compared to these functionals, asymptotically corrected functionals (CAM-B3LYP and LC- $\omega$ PBE) predict larger bindings (by $0.1 \mathrm{eV}$ ), and their calculated $\mathrm{Cd}-\mathrm{Se}$ and $\mathrm{Cd}-\mathrm{O}$ bond lengths agree well with results of high-accuracy CCSD theory applied to small clusters of a few atoms in size. ${ }^{37}$ Nonetheless, all functionals considered predict nearly similar reconstructions of the $\mathrm{QD}$ surface, making the $\mathrm{Cd}-\mathrm{Se}$ bonds longer in the core of the cluster and shorter on the surface, as well as very strong binding (of roughly $1 \mathrm{eV}$ ) between the CdSe cluster and the $\mathrm{OPMe}_{3}$ ligand.

Inclusion of a polar solvent has a dramatic effect on the QD-ligand binding, decreasing the interaction down to $0.2-0.5 \mathrm{eV}$. This is rationalized by the polar character of both QD and ligand structures, whose dipole moments are getting screened by the solvent environment. Consequently, incorporation of the solvent into the calculations is absolutely necessary to obtain realistic QD-ligand interactions comparable with experimental data.

The observed behavior of the energy gaps and the lowestenergy optical transitions with respect to the functionals correlates well with the previously published results for $\mathrm{QDs}^{42,71,72}$ and other nanomaterials. ${ }^{70,73}$ Namely, the pure XC functionals (LSDA and GGA) underestimate the band gaps and do not include relevant excitonic effects in the optical transitions. Hybrid functionals, such as B3LYP and PBE1, provide very reasonable values of the energy gap and transition energies $(\sim 3 \mathrm{eV})$, which correlate well with experimental data. ${ }^{67,68}$ Asymptotically corrected functionals (CAM-B3LYP and LC- $\omega$ PBE) significantly overestimate the HOMO-LUMO gap. However, when Coulombic interactions between photoexcited electrons and holes are included via TD-DFT calculations, the energy of the lowest transition significantly decreases and becomes closer to the experimental values. The effect of the solvent model on the band gap and the lowest optical transition is not as strong as in the case of binding energy, leading to a consistent blue shift of about $0.2-0.3 \mathrm{eV}$, to the respective calculations in the gas phase.

Overall, our studies validate the utilization of the DFT methodology for simulation of the morphology and electronic structure of QDs ligated with organic moieties. However, specific functionals and basis sets have to be carefully chosen for the proper description of the various physical properties of ligated QDs. Presented results provide guidelines in selecting an appropriate modeling tool for predicting the interaction mechanisms and relative interaction strengths between organics and semiconductor nanomaterials.

\section{ASSOCIATED CONTENT}

(5) Supporting Information. Additional Tables and figure. This material is available free of charge via the Internet at http:// pubs.acs.org.

\section{AUTHOR INFORMATION}

\section{Corresponding Author}

*E-mail: svetlana.kilina@ndsu.edu.

\section{ACKNOWLEDGMENT}

This work has been supported by the Center for Advanced Solar Photophysics (CASP), an Energy Frontier Research Center funded by the U.S. Department of Energy (DOE), Office of Science, Office of Basic Energy Sciences (BES). S.K. acknowledges financial support from ND EPSCoR and NSF grant no. EPS-0814442 and DOE start up grant no. DE-FG3608GO88160. S.K. and V.A. thank Ekaterina Badaeva for fruitful discussions. We acknowledge support of Center for Integrated Nanotechnology (CINT) and Center for Nonlinear Studies (CNLS) at Los Alamos National Laboratory. Los Alamos National Laboratory is operated by Los Alamos National Security, LLC, for the National Nuclear Security Administration of the U. S. Department of Energy under contract DE-AC52-06NA25396.

\section{REFERENCES}

(1) Coe, S.; Woo, W. K.; Bawendi, M.; Bulovic, V. Nature 2002, 420, 800-803.

(2) Yoffe, A. D. Adv. Phys. 2001, 50, 1-208.

(3) Koleilat, G. I.; Levina, L.; Shukla, H.; Myrskog, S. H.; Hinds, S.; Pattantyus-Abraham, A. G.; Sargent, E. H. ACS Nano 2008, 2, 833-840.

(4) Gorman, J.; Hasko, D. G.; Williams, D. A. Phys. Rev. Lett. 2005, 95, 090502-1-4.

(5) Schaller, R. D.; Petruska, M. A.; Klimov, V. I. J. Phys. Chem. B 2003, 107, 13765-13768.

(6) Klimov, V. I.; Ivanov, S. A.; Nanda, J.; Achermann, M.; Bezel, I.; McGuire, J. A.; Piryatinski, A. Nature 2007, 447, 441-446.

(7) Dahan, M.; Levi, S.; Luccardini, C.; Rostaing, P.; Riveau, B.; Triller, A. Science 2003, 302, 442-445. 
(8) Michalet, X.; Pinaud, F. F.; Bentolila, L. A.; Tsay, J. M.; Doose, S.; Li, J. J.; Sundaresan, G.; Wu, A. M.; Gambhir, S. S.; Weiss, S. Science 2005, 307, 538-544.

(9) Alivisatos, A. P. Science 1996, 271, 933-937.

(10) Scholes, G. D. Adv. Funct. Mater. 2008, 18, 1157-1172.

(11) Sapra, S.; Nanda, J.; Pietryga, J. M.; Hollingsworth, J. A.; Sarma,

D. D. J. Phys. Chem. B 2006, 110, 15244-15250.

(12) Winkler, U.; Eich, D.; Chen, Z. H.; Fink, R.; Kulkarni, S. K.; Umbach, E. Chem. Phys. Lett. 1999, 306, 95-102.

(13) Green, M. J. Mater. Chem. 2010, 20, 5797-5809.

(14) Chen, Y.; Vela, J.; Htoon, H.; Casson, J. L.; Werder, D. J.; Bussian, D. A.; Klimov, V. I.; Hollingsworth, J. A. J. Am. Chem. Soc. 2008, 130, 5026-5027.

(15) Krauss, T. D.; Peterson, J. J. J. Phys. Chem. Lett. 2010, 1, $1377-1382$.

(16) Nozik, A. J. Annu. Rev. Phys. Chem. 2001, 52, 193-231.

(17) Schaller, R. D.; Petruska, M. A.; Klimov, V. I. Appl. Phys. Lett. 2005, 87, 253102-1-4.

(18) Talapin, D.; Rogach, A.; Kornowski, A.; Haase, M.; Weller, H. Nano Lett. 2001, 1, 207-211.

(19) Guyot-Sionnest, P.; Wehrenberg, B.; Yu, D. J. Chem. Phys. 2005, 123, 074709 .

(20) Liu, I. S.; Lo, H. H.; Chien, C. T.; Lin, Y. Y.; Chen, C. W.; Chen, Y. F.; Su, W. F.; Liou, S. C. J. Mater. Chem. 2008, 18, 675-682.

(21) Cooney, R. R.; Sewall, S. L.; Anderson, K. E. H.; Dias, E. A.; Kambhampati, P. Phys. Rev. Lett. 2007, 98, 177403-1-4.

(22) Guyot-Sionnest, P.; Shim, M.; Matranga, C.; Hines, M. Phys. Rev. B 1999, 60, R2181.

(23) Klimov, V. I.; Mikhailvsky, A. A.; Xu, S.; Malko, A.; Hollingsworth, J. A.; Leatherdale, C. A.; Eisler, M.-J.; Bawendi, M. G. Science 2000, 290, 314-317.

(24) Beard, M. C.; Midgett, A. G.; Law, M.; Semonin, O. E.; Ellingson, R. J.; Nozik, A. J. Nano Lett. 2009, 9, 836-845.

(25) Nair, G.; Bawendi, M. G. Phys. Rev. B 2007, 76, 081304-4.

(26) Ben-Lulu, M.; Mocatta, D.; Bonn, M.; Banin, U.; Ruhman, S. Nano Lett. 2008, 8, 1207-1211.

(27) Nair, G.; Geyer, S. M.; Chang, L.-Y.; Bawendi, M. G. Phys. Rev. B 2008, 78, 125325-10.

(28) McGuire, J. A.; Joo, J.; Pietryga, J. M.; Schaller, R. D.; Klimov, V. I. Acc. Chem. Res. 2008, 41, 1810-1819.

(29) von Holt, B.; Kudera, S.; Weiss, A.; Schrader, T. E.; Manna, L.; Parake, W. J.; Braun, M. J. Mater. Chem. 2008, 18, 27282732.

(30) Pokrant, S.; Whaley, K. B. Eur. Phys. J. D 1999, 6, 255-267.

(31) Frenzel, J.; Joswig, J.-O.; Sarkar, P.; Seifert, G.; Springborg, M. Eur. J. Inorg. Chem. 2005, 18, 3585-3596.

(32) Wang, L. W.; Zunger, A. Phys. Rev. B 1996, 53, 9579-9582.

(33) Reboredo, F. A.; Zunger, A. Phys. Rev. B 2001, 63, 235314235317.

(34) Rabani, E. J. Phys. Chem. C 2009, 113, 1493-1497.

(35) Schapotschnikow, P.; Hommersom, B.; Vlugt, T. J. Phys. Chem. C 2009, 113, 12690-12698.

(36) Nguyen, K. A.; Day, P. N.; Pachter, R. J. Phys. Chem. C 2010, 114, 16197-16209.

(37) Yang, P.; Tretiak, S.; Masunov, A.; Ivanov, S. J. Chem. Phys. 2008, 129, 074709-1-12.

(38) Inerbaev, T. M.; Masunov, A. E.; Khondaker, S. I.; Dobrinescu, A.; Plamada, A.-V.; Kawazoe, Y. J. Chem. Phys. 2009, 131, 044106-1-6.

(39) Chung, S.-Y.; Lee, S.; Liu, C.; Neuhauser, D. J. Phys. Chem. B 2009, 113, 292-301.

(40) Xu, S.; Wang, C.; Cui, Y. J. Mol. Model. 2010, 16, 469-473.

(41) Isborn, C. M.; Kilina, S. V.; Li, X.; Prezhdo, O. V. J. Phys. Chem. C 2008, 112, 18291-18294.

(42) del Puerto, M. L.; Tiago, M. L.; Chelikowsky, J. R. Phys. Rev. Lett. 2006, 97, 096401-096404.

(43) Puzder, A.; Williamson, A. J.; Gygi, F.; Galli, G. Phys. Rev. Lett. 2004, 92, 217401.

(44) Puzder, A.; Williamson, A.; Zaitseva, N.; Galli, G.; Manna, L.; Alivisatos, A. Nano Lett. 2004, 4, 2361-2365.
(45) Rempel, J. Y.; Trout, B. L.; Bawendi, M. G.; Jensen, K. F. J. Phys. Chem. B 2006, 110, 18007-18016.

(46) Kamisaka, H.; Kilina, S. V.; Yamashita, K.; Prezhdo, O. V. J. Phys. Chem. C 2008, 112, 7800-7808.

(47) Kilina, S. V.; Kilin, D. S.; Prezhdo, O. V. ACS Nano 2009, 3, 93-99.

(48) Kilina, S.; Ivanov, S.; Tretiak, S. J. Am. Chem. Soc. 2009, $131,7717-7726$.

(49) Kopping, J. T.; Patten, T. E. J. Am. Chem. Soc. 2008, 130, $5689-5698$

(50) Owen, J. S.; Park, J.; Trudeau, P.; Alivisatos, A. P. J. Am. Chem. Soc. 2008, 130, 12279-12281.

(51) Morris-Cohen, A. J.; Frederick, M. T.; Lilly, G. D.; McArthur, E. A.; Weiss, E. A. J. Phys. Chem. Lett. 2010, 1, 1078-1081.

(52) Wolcott, A.; Fitzmorris, R. C.; Muzaffery, O.; Zhang, J. Z. Chem. Mater. 2010, 22, 2814-2821.

(53) Frisch, M. et al. Gaussian 09; Gaussian, Inc.: Wallingford, CT, 2009.

(54) Sahni, V.; Gruenebaum, J.; Perdew, J. P. Phys. Rev. B 1982, 26, 4371-7.

(55) Perdew, J. P. Electronic Structure of Solids; Ziesche, P., Eschrig, H., Eds.; Akademie Verlag: Berlin, 1991.

(56) Tao, J. M.; Perdew, J. P.; Staroverov, V. N.; Scuseria, G. E. Phys. Rev. Lett. 2003, 91, 146401-4.

(57) Parr, R. G.; Yang, W. Density-Functional Theory of Atoms and Molecules; Oxford University Press: New York, 1989.

(58) Ernzerhof, M.; Perdew, J. P. J. Chem. Phys. 1998, 109, 3313-3320.

(59) Yanai, T.; Tew, D.; Handy, N. Chem. Phys. Lett. 2004, 393, $51-57$.

(60) Tawada, Y.; Tsuneda, T.; Yanagisawa, S.; Yanai, T.; Hirao, K. J. Chem. Phys. 2004, 120, 8425.

(61) Furche, F.; Ahlrichs, R. J. Chem. Phys. 2002, 117, 7433-47.

(62) Vanderbilt, D. Phys. Rev. B 1990, 41, 7892.

(63) Kresse, G.; Furthmüller, J. Phys. Rev. B 1996, 54, 11169.

(64) Barone, V.; Cossi, M.; Tomasi, J. J. Comput. Chem. 1998, 19, 404-417.

(65) Cossi, M.; Rega, N.; Scalmani, G.; Barone, V. J. Comput. Chem. 2003, 24, 669-681.

(66) Botti, S.; Marques, M. A. L. Phys. Rev. B 2007, 75, 035311.

(67) Kasuya, A.; Sivamohan, R.; Barnakov, Y. A.; Dmitruk, I. M.; Nirasawa, T.; Romanyuk, V. R.; Kumar, V.; Mamykin, S. V.; Tohji, K.; Jeyadevan, B.; Shinoda, K.; Kudo, T.; Terasaki, O.; Liu, Z.; Belosludov, R. V.; Sundararajan, V.; Kawazoe, Y. Nat. Mater. 2004, 3, 99-102.

(68) Kudera, S.; Zanella, M.; Giannini, C.; Rizzo, A.; Li, Y.; Gigli, G.; Cingolani, R.; Ciccarella, G.; Spahl, W.; Parak, W. J.; Manna, L. Adv. Mater. 2007, 19, 548-552.

(69) Shim, M.; Guyot-Sionnest, P. J. Chem. Phys. 1999, 111, 69556964.

(70) Tretiak, S.; Igumenshchev, K.; Chernyak, V. Phys. Rev. B 2005, 71,33201

(71) Izmaylov, A. F.; Scuseria, G. E. J. Chem. Phys. 2008, 129, 034101-10.

(72) Onida, G.; Reining, L.; Rubio, A. Rev. Mod. Phys. 2002, 74, $601-659$.

(73) Kilina, S.; Badaeva, E.; Piryatinski, A.; Tretiak, S.; Saxena, A.; Bishop, A. R. Phys. Chem. Chem. Phys. 2009, 11, 4113-4123. 